Synthesis, vol. 25 n 1 , e032, junio 2018. ISSN 1851-779X

Universidad Nacional de La Plata.

Facultad de Humanidades y Ciencias de la Educación.

Synthesis

Centro de Estudios Helénicos

\title{
¿Cómo elevarse a lo divino? Sobre las últimas palabras de Plotino
}

\author{
José María Zamora Calvo * \\ * Universidad Autónoma de Madrid, España
}

Cita sugerida: Zamora Calvo, J. M. (2018). ¿Cómo elevarse a lo divino? Sobre las últimas palabras de Plotino. Synthesis, 25(1), e032. https://doi.org/10.24215/1851779Xe032 


\title{
¿Cómo elevarse a lo divino? Sobre las últimas palabras de Plotino
}

How to Rise towards the Divine? On Plotinus' Last Words

\author{
José Maria Zamora Calvo * \\ Universidad Autónoma de Madrid, España
}

\section{RESUMEN:}

Las últimas palabras pronunciadas por Plotino a su médico y discípulo Eustoquio (V.P. 2.25-27) constituyen una exhortación a la filosofía, expresada a modo de último consejo, que conecta con el tema platónico del ascenso y regreso del alma. Es preciso huir del mundo de acá hacia el de allá, para regresar, como Odiseo, a la verdadera patria. En la arquitectura plotiniana las tres hipóstasis divinas, además de ser constitutivas de la estructura de la realidad transcendente, se hallan también de manera latente presentes en el hombre. ¿Cómo explicar en palabras ese esfuerzo por hacer elevar lo que hay de divino en nosotros a lo que hay de divino en el universo? Más allá de Eustoquio, el único destinatario inmediato, esta exhortación-recomendación se extiende a toda la humanidad.

Palabras clave: Vida de Plotino, Porfirio, alma, lo divino, anagogé.

\section{Abstract:}

Plotinus' last words uttered to his physician and disciple Eustochius (V.P. 2.25-27) are an exhortation to philosophy, expressed as a last piece of advice, which connects with the Platonic theme of the ascent and return of the soul. It is necessary to flee from the world here to the world over there, to return, like Odysseus, to the true homeland. In the Plotinian architecture, the three divine hypostases as well as constituting the structure of the transcendent reality are also latently present in man. How to explain in words that effort to elevate what is divine in us to what is divine in the universe? Beyond Eustochius, the only immediate recipient, this exhortation-recommendation extends to all humanity.

KEYWORDS: Plotinus' life, Porphyry, soul, the divine, anagoge.

In Memoriam Zeke Mazur

A comienzos del segundo año del reinado de Claudio, es decir, durante el otoño o invierno de 269, Plotino deja Roma y se traslada a Campania, donde redacta sus últimos cuatro tratados (Porfirio, V.P. 6.16-25). Tras varios meses retirado en la finca, propiedad de su antiguo compañero ya fallecido Zeto, ${ }^{1}$ Plotino convalece en soledad, sufriendo una especie de tuberculosis o de gangrena, ${ }^{2}$ apartado de sus amigos y discípulos, sin poder escribir ni dictar. De los más allegados integrantes de su círculo romano, ${ }^{3}$ cuando estaba instalado enseñando en casa de Gémina (9.2), unos habían ya fallecido, como el propio Zeto, Zótico y Paulino (2.9-20; 7.15-17), y otros habían partido, Porfirio casi dos años antes a Sicilia y Amelio un año después a Apamea, en Siria (2.31-33; 6.1-3), al parecer por cierta repulsión a la enfermedad que padecía su maestro (2.15-17) y probablemente también por miedo al contagio. Las cosas necesarias para su día a día provenían de las propiedades de Zeto, además de la hacienda de Castricio, a quien Porfirio dedicará su tratado Sobre la abstinencia, que tenía sus posesiones en Minturnas (2.21-23). En el verano de 270, Plotino fallece, a la edad de sesenta y seis años. ${ }^{4}$ En sus últimos momentos, solo Eustoquio, su médico y discípulo fiel, vino a verlo cuando yacía moribundo, trasladándose desde su residencia en Putéolos hasta Minturnas, llegando justo a tiempo para escuchar las últimas palabras del maestro.

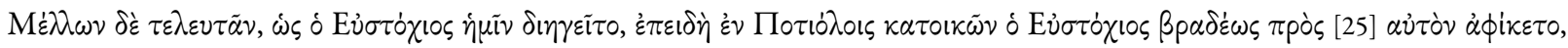

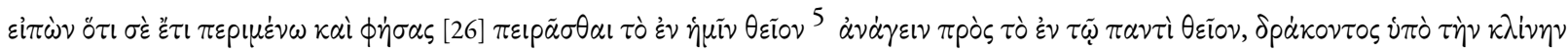

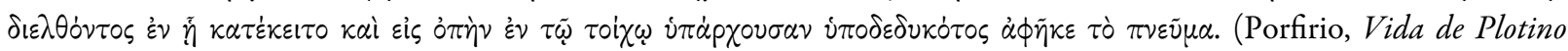
2.23-29 H-S ${ }^{1}$ )

Cuando [Plotino] estaba a punto de morir, según nos contaba Eustoquio, una vez que Eustoquio, que vivía entonces en Putéolos, llegó a su lado con retraso, Plotino le dijo: 'A ti te estoy esperando aún'. Y tras haberle recomendado que se esforzara 
por hacer elevar lo que de divino hay en nosotros hacia lo que hay de divino en el universo, en el momento en que una serpiente se deslizó por debajo del lecho en que yacía aquel y se coló en una hendidura que había en la pared, exhaló su hálito.

\section{Sumario De UnA Vexata guaestio}

El pasaje de las últimas palabras atribuidas a Plotino en su lecho de muerte es considerado por Most (2003: 576) uno "de los más controvertidos en la literatura griega posterior". De hecho, el debate filológico que desencadena sigue siendo insoluble, si nos atenemos a las diferentes familias de manuscritos que transmiten

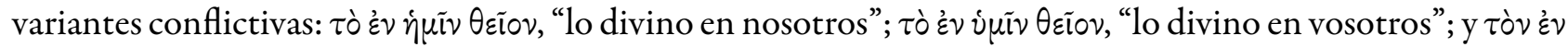
ن

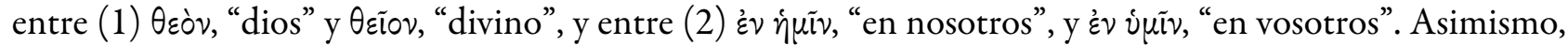
tal como cabe esperar, esta controversia filológica repercute con firmeza en las concepciones filosóficas sobre la inmortalidad del alma, la teología y la cosmología plotinianas.

Podemos sintetizar en la siguiente tabla las principales interpretaciones discrepantes, que comienzan a partir de la línea 26, basadas en una particular lectura del texto, en la gramática y en la exégesis específica del pasaje:

\begin{tabular}{|c|c|c|}
\hline & 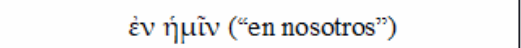 & ċv ú $\mu \tilde{v}$ ("en vosotros") \\
\hline$\theta \varepsilon o ̀ v$ ("dios") & $\begin{array}{l}\text { Schwyzer 1976; } \\
\text { Most } 2003\end{array}$ & $\begin{array}{l}\text { H-S } 1964 \text { (editio minor); } \\
\text { Henry 1953; Sala } 2002\end{array}$ \\
\hline$\theta \varepsilon$ ĩov ("divino") & $\begin{array}{l}\text { H-S } 1951 \text { (editio maior); } \\
\text { Harder 1958; Igal 1972; } \\
\text { Pépin 1992; D'Ancona Costa } \\
\text { 2002; Mazur } 2017\end{array}$ & \\
\hline
\end{tabular}

Desde 1953, los filólogos, comentaristas e historiadores que se han dedicado a examinar el pasaje de Porfirio que contiene los ultima verba de Plotino han considerado tres posibles destinatarios: el círculo que integra a todos sus discípulos (Henry); exclusivamente el médico Eustoquio, el único discípulo presente (Igal); o toda la humanidad (Schwyzer).

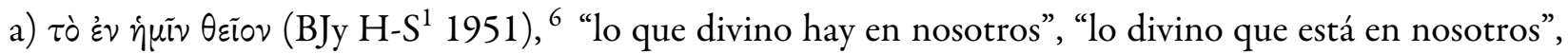
considerada desde el Renacimiento, a excepción de Perna (1580), la interpretación clásica o tradicional, analiza $\phi \dot{\eta} \sigma \alpha \varsigma \pi \varepsilon ı \tilde{\rho} \sigma \theta \alpha \iota$ como una construcción en estilo indirecto con un verbum dicendi seguido de un infinitivo enunciativo. Según esta lectura, las últimas palabras de Plotino incluyen una declaración reservada de carácter autobiográfico que, expresada en estilo directo, podría enunciarse del siguiente modo: "Estoy esforzándome por hacer elevar lo que de divino hay en nosotros a lo que hay de divino en el universo".

El texto de esta interpretación aparece en todas las ediciones posteriores a la editio princeps de Perna, publicada en Basilea (1580), y anteriores al artículo de Henry (1953). La fórmula del Timeo (90c), unida

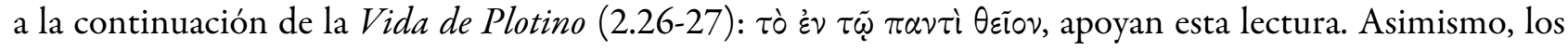
traductores de este pasaje anteriores a Henry adoptan este texto, escogiendo entre dos posibles variantes:

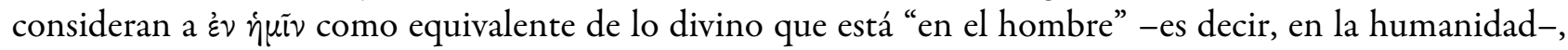
o como un plural mayestático, que corresponde a lo divino que está "en mí". Posteriormente al artículo de Henry, esta interpretación tradicional ha sido aceptada y defendida por Dodds (1964), Harder (1958), Pépin (1992) y, más recientemente, por D’Ancona Costa (2002) y Mazur (2017).

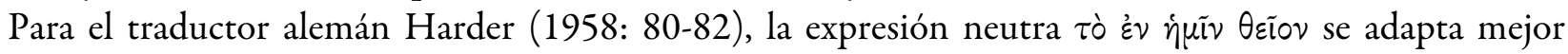

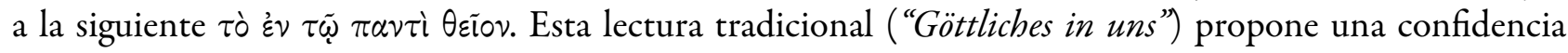
autobiográfica de Plotino, que contiene una fórmula casi técnica, heredera de una rica tradición filosófica. ${ }^{7}$ 
De este modo, lo divino presenta dos estados, inmanente en el hombre y transcendente en el universo, que se unifican en la muerte.

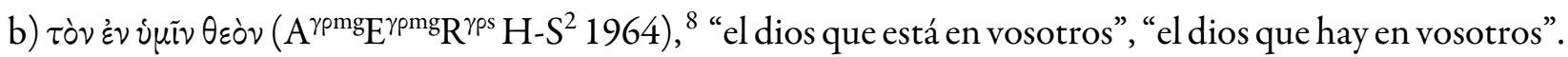
Henry (1953) aporta una nueva solución a este problema paleográfico, adoptando una variante que aparece ya en la edición basilense de Perna (1580), ${ }^{9}$ que será la incorporada finalmente en la editio minor (H-S ${ }^{2}$ 1964), ${ }^{10}$ y que ha sido aceptada por Armstrong (1966) en su traducción inglesa, ${ }^{11}$ por Beierwaltes (1967: 193-194), para sustentar su traducción alemana y comentario de un pasaje correspondiente al tratado Sobre la eternidad y el tiempo (En. III.7 [37] 5.18-19), y, más recientemente, apoyada por Sala (2002).

Esta interpretación considera $\phi \dot{\eta} \sigma \alpha \varsigma$ como un participio que introduce las palabras textuales de Plotino en estilo directo y $\pi \varepsilon \iota p \tilde{\alpha} \sigma \theta \alpha \iota$ como un infinitivo en función de un presente de imperativo de segunda persona del plural. Según esta lectura, las últimas palabras de Plotino no constituyen una declaración autobiográfica, sino un último consejo del maestro dirigido a sus discípulos: "Esforzaos por hacer elevar el dios que hay en vosotros a lo que hay de divino en el universo". ${ }^{12}$

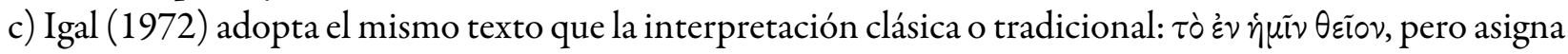
a $\phi \dot{\eta} \sigma \alpha \varsigma \pi \varepsilon ı \tilde{\alpha} \sigma \theta \alpha \mathrm{l}$ un sentido gramatical diferente. Según esta lectura, se trata de una construcción en estilo indirecto, pero analiza $\phi \dot{\gamma} \sigma \alpha \varsigma$ como un verbum dicendi equivalente a un verbum iubendi, con un carácter exhortativo. El sujeto del participio $\phi \dot{\eta} \sigma \alpha \varsigma$ es Plotino, mientras que el del infinitivo $\pi \varepsilon \iota \tilde{p} \sigma \sigma \alpha \iota$ es Eustoquio. De este modo, las últimas palabras de Plotino, expresadas en estilo directo, mediante un imperativo presente de segunda persona del singular $(\pi \varepsilon \uparrow \tilde{p} \tilde{)})$, podrían traducirse así: "Esfuérzate por reconducir lo que de divino hay en nosotros a lo que hay de divino en el universo".

La interpretación de Igal resulta convincente, en la medida que pretende conciliar las otras dos, tratando de conservar sus méritos y de reducir lo más posible sus desventajas: el texto mantiene la lectura tradicional, pero en su exégesis añade un sentido protréptico o exhortativo que la aproxima a la propuesta de Henry, aunque sin coincidir plenamente con ella. Henry confiere a las últimas palabras de Plotino el carácter de una última recomendación del maestro a todos sus discípulos, mientras que en la interpretación de Igal, la recomendación, a modo de consigna, va dirigida exclusivamente al único discípulo presente, su médico Eustoquio.

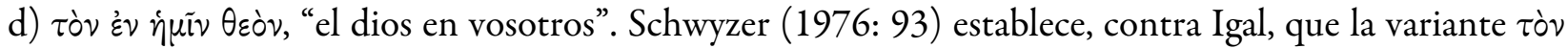
[...] $\theta$ còv no constituye una alteración cristiana. Asimismo, aunque sea la lectio difficilior, el editor suizo la considera correcta. Una solución ante la que Most (2003: 583-584) no se muestra en desacuerdo. Si la buena

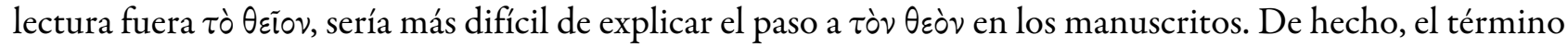
$\theta$ eós lo utiliza con frecuencia Plotino para designar la inteligencia o el alma en el hombre, en contraste con el neutro $\theta$ عĩov.

Por otra parte, para Schwyzer (1976: 96) el destinatario de la exhortación a quien dirige sus últimas palabras Plotino es toda la humanidad. Pero, a diferencia de Henry (1953), Schwyzer recurre a la corrección introducida por de Strycker: $\pi \varepsilon i p \tilde{\alpha} \sigma \theta \varepsilon$ en lugar de $\pi \varepsilon i p \tilde{\sigma} \sigma \theta \alpha \mathrm{l}$ (infinitivus pro imperativo). Aunque el grupo

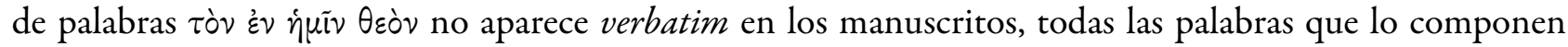
sí están recogidas en ellos. No obstante, ante el problema de la veracidad del relato, Schwyzer (1976: 97) aboga por la prudencia: probablemente, de manera semejante a lo sucedido con el oráculo de Apolo, sus discípulos modelaron las palabras finales en forma de leyenda para ensalzar así la figura del maestro fundador del neoplatonismo. ${ }^{13}$

\section{ConteXto DRAmático}

Dado que se encontraba viviendo en Lilibeo (Sicilia), para el relato de las últimas palabras de su maestro, Porfirio se basa solamente en el testimonio de Eustoquio, discípulo y médico de cabecera de Plotino, quien 
cuida de él hasta sus últimos días, acompañándolo en el momento de su muerte (V.P. 2.12): "Asimismo otro médico, el alejandrino Eustoquio, el cual, dándose a conocer en la última etapa de su vida, perseveró con él

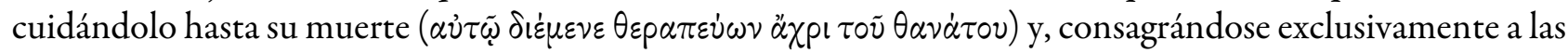

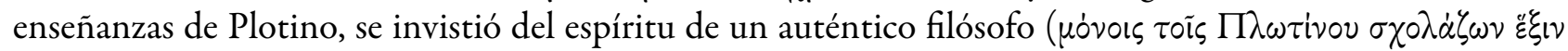

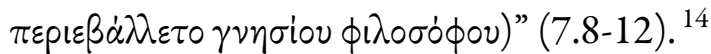

Testigo excepcional de la muerte de Plotino, Eustoquio fue el único de los discípulos que estaba presente junto a su maestro (V.P. 2.34), si bien llega con retraso a la villa de Zeto, porque vivía en la ciudad de Putéolos (actualmente Pozzuoli), situada en la costa de Campania, a doce kilómetros de Nápoles. La distancia entre Miturnas (actualmente Miturno), donde tenía sus posesiones Castricio Firmo, y Putéolos es de sesenta kilómetros. Podemos pensar, como sugiere Brisson (2010: 319, n. 19), que Eustoquio se desplaza en barco desde Putéolos hasta Miturnas, ciudad con puerto cercana a la villa en la que se había retirado Plotino.

La escena de la serpiente retoma un tema mitológico ampliamente atestiguado en la tradición griega que compara el alma humana, separada del cuerpo, con una serpiente, el animal ctónico por antonomasia, o con un pájaro, el animal celeste por excelencia. ${ }^{15}$ Con forma de serpiente, el alma surge de la tierra y a ella regresa, como la que se deslizó por debajo del lecho en que yacía Plotino y se escabulló por un hueco que había en el muro (V.P. 2.27-28). Asimismo, como animal sagrado o cercano a la divinidad, se rinde culto a la serpiente, forma bajo la cual se manifiesta Asclepio. ${ }^{16}$ Según relatan diversos autores, este mismo reptil surge de la médula de un hombre muerto. ${ }^{17}$

La muerte de Plotino se representa con la entrega del hálito $(\pi \nu \varepsilon \tilde{\nu} \mu \alpha)$ (V.P. 2.29): cuando alguien exhala o entrega el soplo vital, muere. ${ }^{18}$ Tras su muerte, su discípulo y ayudante Amelio preguntó a Apolo adónde había ido el alma de su maestro. El dios délfico le habría respondido en forma de oráculo-himno, compuesto por 51 hexámetros dactílicos, que Porfirio recopila (V.P. 22) y comenta (V.P. 23). Según proclama el oráculo de Delfos, el alma de Plotino, separada del cuerpo, se halla ahora en el cielo con los dioses y los démones. ${ }^{19}$

\section{El testimonio de Sinesio}

Sinesio de Cirene (ca.370-413), que llegó a ser obispo de la Pentápolis libia, no ejerce la profesión de filósofo como los profesores y comentadores que imparten sus enseñanzas en las escuelas filosóficas de fines de la Antigüedad, pero vive de una manera filosófica siguiendo su propia vocación. ${ }^{20}$ La colección de 156 cartas, que revela datos sobre su propia vida, destaca por la variedad temática y la elegancia retórica. Para Bregman (2010: 523), estas epístolas, que incluyen varias remitidas a su maestra Hipatia de Alejandría, "pasaron de comunicaciones personales y familiares a discusiones filosóficas". De los más de cuarenta destinatarios, su hermano Evoptio es el más habitual, de una correspondencia redactada desde la última década del s. IV hasta el mismo año de su muerte. ${ }^{21}$

A su antiguo condiscípulo Herculanio le remite diez cartas (137-146), desde Cirene a Alejandría, entre los años 393 y 399. En esta correspondencia, profundamente influida por las enseñanzas neoplatónicas, Sinesio celebra la amistad filosófica que le une a Herculanio y concluye más de una vez con una exhortación a la filosofía, para que se aparte de la muchedumbre y se refugie en el sosiego de la intimidad (Ep. 137). Pero en la carta 139, datada en el primer semestre de 398, para exhortar a su amigo, Sinesio recurre, como él mismo reconoce, a las últimas palabras de Plotino. De este modo, si Plotino fallece en el año 270 y Porfirio escribe su biografía en el año 300, Sinesio redacta sus cartas a menos de un siglo de la Vida de Plotino:

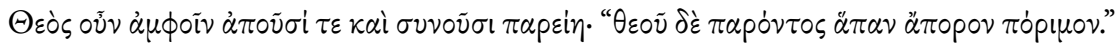

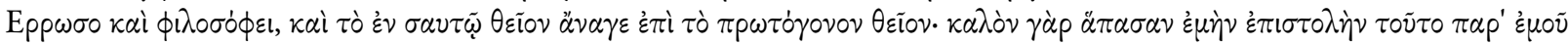

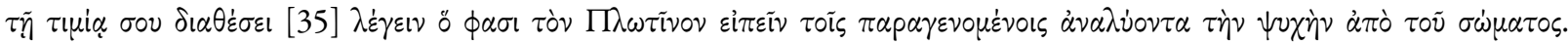
(Sinesio, Epistola 139.30-37) 
Sea como sea, ya estemos juntos o lejos el uno del otro, ¡que Dios esté a nuestro lado! Que, cuando Dios está al lado 'todo lo imposible es posible'. Que sigas bien y dedicado a la filosofía, y 'lo divino que hay en nosotros haz que se eleve hacia lo divino que fue engendrado en primer lugar'. Sí, bella cosa es que mi carta entera exprese de mi parte a tu honrada distinción lo que afirman que Plotino les dijo a los presentes, a punto de despedir ya a su alma del cuerpo. (Trad. García Romero, 1995: 264-265)

Sinesio altera sutilmente el texto de la Vida de Plotino que transmite los ultima verba del maestro de

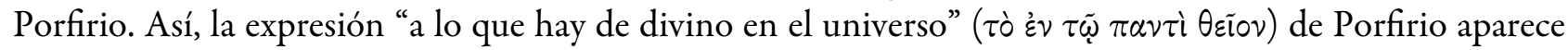
modificada en esta carta dirigida a Herculanio por "hacia lo divino que fue engendrado en primer lugar

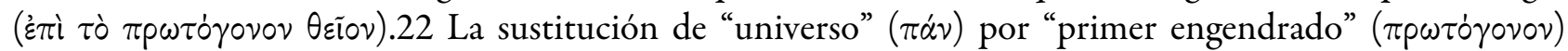
plantea, como señala Pépin (1992: 357, n. 5), dificultades semánticas, derivadas de la lectio que aportan la mayoría de los manuscritos. Es posible adoptar "primer procreador" ( $\pi \rho \omega \tau$ ó $о \vee \vee \nu)$, siguiendo a h [consensus codicum MP], o "progenitor" ( $\pi$ póyovov), variante de x [consensus codicum A(AlPb)AvC(Ur)ILUVVa(et Ang Patm.)]. Ahora bien, si mantenemos $\pi p \omega \tau \dot{y}$ yovov, conectándolo con el texto de Porfirio, el "primer engendrado", es decir, "el que fue engendrado en primer lugar" puede referirse al alma del mundo que el alma individual de cada uno, 23 concretamente la de Herculanio a quien va remitida la carta, quiere imitar. Para alcanzar la Inteligencia divina, primeramente la inteligencia humana individual ha de unirse a la Inteligencia del universo.24

Por otra parte, según Pépin (1992: 376), la carta de Sinesio recoge, probablemente de "segunda mano", "lo que se dice" o "lo que afirman" (o $\phi \alpha \sigma l)$ que fueron las últimas palabras de Plotino que "les dijo a

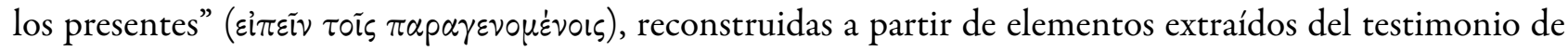
Porfirio sobre la muerte del maestro. Sin embargo, hay piezas que no encajan muy bien en el relato de Sinesio. Porfirio, por ejemplo, únicamente menciona la presencia de Eustoquio, su médico y fiel discípulo, que llega a

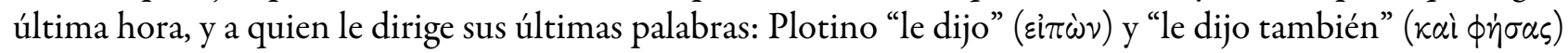
(V.P. 2.25). En el momento de su fallecimiento Porfirio señala que "estaba presente Eustoquio solo" ( $\mu$ óvos $\delta \dot{\varepsilon}$ $\pi \alpha \rho \tilde{\eta} \nu \delta \dot{~ E u ̉ \sigma \tau o ́ \chi ı \varsigma \varsigma, ~ 2.34), ~ s i n ~ e s t a r ~ a c o m p a n ̃ a d o, ~ c o m o ~ r e l a t a ~ S i n e s i o, ~ p o r ~ u n ~ g r u p o ~ d e ~ d i s c i ́ p u l o s ~ o ~ a l l e g a d o s, ~}$ pues el propio biógrafo de Tiro, como ya hemos indicado, se encontraba viviendo en Lilibeo, Amelio en Apamea de Siria y Castricio en Roma (V.P. 2.31-33). Ante ambos testimonios, nos hallamos ante piezas que coinciden, y otras que aparecen distorsionadas, adaptadas por el obispo neoplatónico al contexto de una epístola que pretende exhortar a la filosofía a su destinatario Herculanio.

\section{Elevación a LO Divino}

Las últimas palabras atribuidas a Plotino presentan un carácter eminentemente protréptico, ya que exhortan a hacer elevar el alma, en el sentido de un ascenso y un retorno al origen del que procede. En un primer momento, estas palabras van dirigidas a Eustoquio, ciertamente el único discípulo presente que le acompaña, aunque de un modo semejante a la teoría de los círculos concéntricos propuesta por el estoico Hierocles

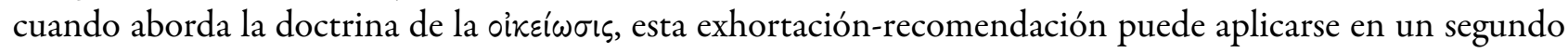
momento a toda la humanidad. ${ }^{25}$

En efecto, la lectura de las Enéadas exhorta a la práctica de la $\alpha \nu \alpha \gamma \omega \gamma \eta \dot{\eta}$, es decir, la "elevación” y "retorno" del alma a los principios de los que procede. Los dos sentidos fundamentales del verbo $\alpha \dot{\alpha} \alpha \omega \omega$ son el de "reconducir", "devolver", "restituir" y el de "elevar", "levantar", "alzar", "remontar a lo alto", "hacer subir", y ambos están presentes en la noción plotiniana de $\alpha \nu \alpha \gamma \omega \gamma \eta$, que es a la vez una "subida” y un "retorno”, es decir, una elevación y una devolución o reconducción.

Plotino emplea el término $\alpha \nu \alpha \gamma \omega \gamma \eta \dot{~ d e l ~ s i g u i e n t e ~ m o d o: ~}{ }^{26}$ "Si hay algo a continuación del Primero, es necesario que o provenga inmediatamente ( $(\dot{v} \theta \dot{v} \varsigma)$ de él o se remonte ( $\alpha \nu \alpha \gamma \omega \gamma \eta \dot{\nu})$ hasta él a través de los intermediarios, y que haya un orden entre los seres de segundo y de tercer rango, en que el segundo se remonte al primero y el tercero al segundo" (En. V.4 [7] 1.1-4). Plotino establece con precisión que, a continuación 
del Primero (el primer principio, el Uno, el Bien), solo hay dos seres, o dos clases de seres (la Inteligencia, que es el segundo, y el Alma, que es el tercero) (En. V.1 [10] 8.1-4).

En la Sentencia 13, Porfirio sostiene que las realidades superiores generan las realidades inferiores. Entre las realidades engendradas, algunas se vuelven hacia su generador, y otras no. El alma, que es un ser de tercer rango, se halla en una situación intermedia, lo que fundamenta las cuestiones éticas. Si, por naturaleza, el alma ha de inclinarse hacia las realidades inferiores que ella misma ha producido, los cuerpos y la materia, la falta para ella radica en esta inclinación, donde la materia se identifica entonces con un mal. Pero Porfirio contrapone el apego al cuerpo a la conversión ( $\dot{\pi} \iota \sigma \tau \rho \circ \phi \eta ́)$ de tipo moral que vincula el alma humana a su realidad generadora, la inteligencia (Sent. 32.51-53). Por ello, esta conversión del alma hacia la inteligencia es de orden moral, ya que sin ella no puede alcanzarse la felicidad, dado que el "retorno" hacia la inteligencia implica un intento de suprimir una inclinación excesiva hacia el cuerpo.

Según expone Porfirio en la Sentencia 30, entre las realidades universales y perfectas, ninguna se halla vuelta hacia lo que ella misma ha engendrado, sino que, por el contrario, todas son elevadas hacia las realidades que las han engendrado. ${ }^{27}$ Así, el cuerpo del mundo, al ser perfecto, se ha elevado hacia el alma que está dotada de inteligencia; el alma del mundo se ha elevado hacia la Inteligencia, y la Inteligencia hacia el Primero (Sent. 30.1-10). En cambio, en las realidades particulares se da la posibilidad de que se inclinen hacia lo que han engendrado, de ahí proviene la posibilidad de la falta $(\dot{\alpha} \mu \alpha \rho \tau i ́ \alpha) .{ }^{28}$ Para estas realidades inferiores la materia es un mal, ${ }^{29}$ ya que pueden volverse hacia ella, teniendo la posibilidad de convertirse a las realidades superiores de las que proceden (30.11-19).

Con el mito del adolescente Narciso, Plotino explica que la belleza corpórea constituye la imagen de la belleza superior e inteligible. Frente a Narciso, atrapado por su reflejo en el espejo de las aguas, símbolo del mundo sensible, se halla Odiseo, quien logra huir de los placeres sensibles proporcionados por la maga Circe y Calipso. El alma "narcisista", como le sucedía a Elpénor -el más joven entre los compañeros de Ulises (Odisea 10.550-560)-, sucumbe porque ignora que el cuerpo es tan solo un reflejo de sí misma. Por el contrario, el alma "odiseica" capta que el cuerpo es simplemente un reflejo de sí misma, y se vuelve hacia una realidad verdadera y anterior a ella de la que procede. Por tanto, esta alma "odiseica" huye del mundo de acá al de allá que constituye su verdadera patria. Pero, ¿en qué consiste esta huida ( $\phi v \gamma \dot{\eta})$ ? y ¿cómo se consigue? Esta huida es un entrar en sí mismo, lo que equivale a regresar a la verdadera patria, pero siguiendo dos etapas: en la primera se dirige del mundo sensible al inteligible, simbolizado en el mito por los placeres de la vista y la belleza sensible; y, en una segunda etapa, se va de lo inteligible al Uno-Bien, simbolizado por "nuestro padre" (En. I.3 [30] 1.11-18). ${ }^{30}$

No obstante, para llevar a cabo esta subida ( $\dot{\alpha} \nu \alpha \gamma \gamma \gamma \dot{\eta})$, el alma "odiseica" no necesita salir de sí misma, pues las tres hipóstasis -el Alma, la Inteligencia y el Uno-Bien- que remonta de modo escalonado, además de ser constitutivas de la estructura jerárquica de la realidad transcendente, se encuentran presentes en el interior del alma humana (En. I.1 [53] 8.1-12; V.1 [10] 1-6). De este modo, el alma capta el Uno-Bien con lo que de ella hay de semejante a él, ya que en ella hay presente algo del primer principio, lleva consigo algo de él que no es del todo inteligencia, sino que está por encima de ella (En. VI.8 [39] 15.14-21; VI.9 [9] 8.19-20). El alma capta con su "propio centro", que también es el centro de la tercera hipóstasis, el principio del que procede, la Inteligencia, y de ahí asciende al Uno-Bien.

Por tanto, en el alma están presentes de modo activo las tres hipóstasis, pero ha de ser la propia alma la encargada de activar y actualizar esa presencia. Precisamente, las últimas palabras que Plotino pronuncia en la villa de Zeto constituyen una prueba de esta actualización, según nos transmite Eustoquio. Estas últimas palabras atribuidas a Plotino constituyen, como afirma Igal (1982: 23), "una síntesis de su propia filosofía". En efecto, muchos de los temas desarrollados en las Enéadas se hallan aquí concentrados en tres líneas. El tema de la huida y subida del alma, recogido de Platón, simboliza la huida que efectúa Odiseo del mundo de acá al de allá y su retorno a la verdadera patria. La fuente de la que parte es la alegoría de la caverna en el libro VII de la República, donde enlaza el ascenso y contemplación de las cosas de arriba con la "subida del 


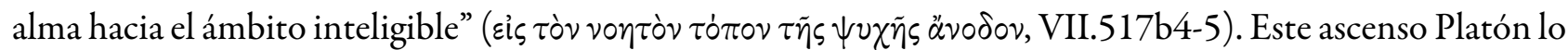
compara con algunos héroes que "han ascendido $(\dot{\alpha} \nu \varepsilon \lambda \theta \varepsilon i v)$ desde el Hades hasta los dioses" (VII.521c2-3). El pasaje de la anagogé del héroe nos recuerda el retorno de Odiseo a Ítaca, su verdadera patria, por lo que puede ponerse en conexión con la anábasis que lleva a cabo el prisionero de la caverna. De este modo, las últimas palabras de Plotino pueden enlazarse con el regreso de Odiseo y la huida del prisionero de la caverna. El método dialéctico "empuja poco a poco al ojo del alma $(\psi v \chi \tilde{\eta} s ~ o ̋ \mu \mu \alpha)$, cuando está sumergido realmente en el fango de la ignorancia, y lo eleva a las alturas ( $\dot{\alpha} \nu \dot{\alpha} \gamma \varepsilon\llcorner\dot{\alpha} \nu \omega)$ )" (VII.533d1-3). Desde una perspectiva escatológica, según nos propone Pépin (1992: 365-366), este pasaje sugiere la alianza entre la muerte y el ascenso del alma. En el momento que el alma se libera del cuerpo, Plotino puede conseguir lo que estaba aguardando: elevar $(\dot{\alpha} \nu \alpha ́ \gamma \omega)$ lo que de divino hay en el alma hacia lo que hay de divino en el universo.

\section{LA BELlezA DEL ALMA PURIFiCADA, SUBidA A LA INTELIGENCIA, SE ASEMEJA A Dios}

Podemos considerar que "lo que de divino hay en nosotros" corresponde al alma de Plotino. Según esto, las últimas palabras aluden al esfuerzo por reconducir su alma a "lo que hay de divino en el universo", es decir, al Alma total o universal $\left(\pi \tilde{\alpha} \sigma \alpha \psi \gamma \chi \eta^{\prime}\right) .{ }^{31}$ De este modo, el Alma total o universal es el origen del que proceden las demás almas -las almas particulares-, y el término adonde bajan, y al que también pueden remontar $(\dot{\alpha} \nu \varepsilon \lambda \theta \varepsilon \tilde{\varepsilon} \nu)$. Pero el Alma total está por naturaleza siempre arriba ( $\dot{\alpha} \varepsilon \dot{\alpha} \alpha \omega, E n$. III.9 [13] 3.1-7).

El alma, que ocupa una posición intermedia entre lo inteligible y lo sensible, ${ }^{32}$ puede proyectar los lógoi en la materia, pero también, en vez de permanecer junto al Alma total, se ve arrastrada por su anhelo de independencia y penetra en los objetos sensibles (En. IV.8 [6] 7.1-14). Una vez que se ha purificado, el alma se vuelve idea, razón totalmente incorpórea e intelectiva (En. I.6 [1] 6.13-14). Ahora bien, el hecho de que el alma pueda volverse "totalmente incorpórea" ( $\pi \dot{\alpha} \nu \tau \eta \dot{\alpha} \sigma \dot{\omega} \mu \alpha \tau \circ \varsigma)$ plantea la dificultad de que antes de suceder esto no era del todo incorpórea, y que hasta que no ha remontado totalmente hacia la Inteligencia (6.16) no

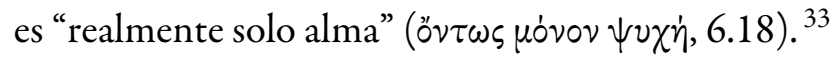

Precisamente, la belleza del alma radica en la vinculación del alma con la realidad inteligible, es decir, en su conexión con las formas inteligibles. ${ }^{34}$ De este modo, el ser inteligible produce la belleza en el alma, quien a su vez la transmite al mundo sensible. Por ello, la realidad inteligible es la belleza primordial. ${ }^{35}$ La Inteligencia y el lógos son la forma propia del alma, que hacen al alma plenamente alma. ${ }^{36}$

El alma se integra toda ella en lo divino (En. I.6 [1] 6.14-15). Tanto el alma como la Inteligencia puede decirse que son divinas. Ahora bien, Plotino establece una distinción entre "integrarse" o "pertenecer a lo divino" y el nivel supraintelectivo del Uno-Bien que se identifica con "Dios" (6.20). Asimismo, en Dios son

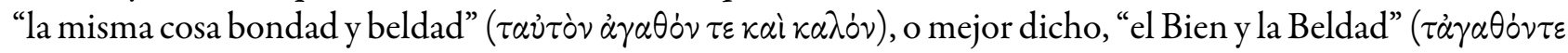

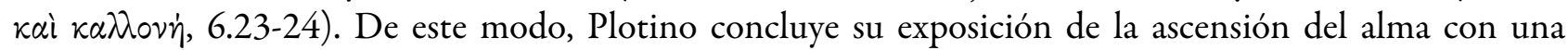
referencia implícita a Dios y la divinidad del alma. Para el alma, el hacerse buena y bella consiste "en asemejarse

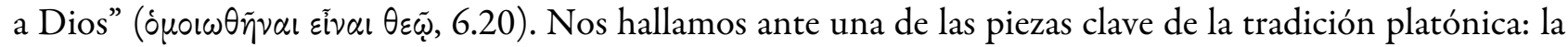
$\delta \mu o i \omega \sigma \iota \varsigma \tau \tilde{\omega} \theta \varepsilon \tilde{\omega}$ es un concepto fundamental en el Teeteto (176a-b) y en el Timeo (90d), donde se proclama que el objetivo último humano es la imitación del dios, entendida como la adecuación de las revoluciones del hombre a las del mundo. Este sería el cometido de la física del Timeo: un procedimiento para la asimilación con lo divino a través de la imitación de la armonía y la mesura presente en la génesis del cosmos. ${ }^{37} \mathrm{El}$ fin de la filosofía es aproximarse al dios lo más posible o, en el mejor de los casos, lograr una semejanza plena con él. ${ }^{38}$

En este pasaje del tratado Sobre la belleza (En. I.6 [1] 6.20-21), Dios, que es la fuente de la que nacen la

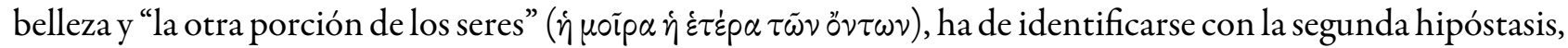
la Inteligencia, más que con el Uno-Bien. La belleza sería una forma determinada en el interior del ámbito inteligible, que coexiste con las otras formas, es decir, con "la otra porción de los seres". ${ }^{39}$ 
Plotino hereda de Platón el principio del conocimiento de "lo semejante por lo semejante". Así, el vidente, para poder ver en la contemplación, ha de purificarse de los vicios y, de este modo, obtener la energía que le faltaba para poder mirar las cosas muy brillantes. ${ }^{40} \mathrm{El}$ conocimiento no sería posible sin esta "afinidad" o "semejanza" entre el conocedor y lo conocido, entre el sujeto vidente y el objeto de la visión (En. I.6 [1] 9.29-32). Cuando el alma ha llegado a la belleza de la Inteligencia (En. VI.7 [38] 16.15-16), es el momento de ver, no de discurrir.

El ojo es afin al sol en Platón, y el alma, para ver la belleza, ha de hacerse bella. ${ }^{41}$ Pero Platón no menciona un nivel supraintelectivo en el alma, como lo hace Plotino, quien lleva hasta sus últimas consecuencias el principio del conocimiento de "lo semejante por lo semejante" y lo aplica, más allá de la segunda hipóstasis, a la visión del Uno-Bien (En. VI.9 [9] 11.30-32). El centro del alma es también el principio del alma y es semejante al primer principio. ${ }^{42}$ En el fondo no se trata de percibir visualmente un objeto, sino de hacer coincidir el centro del alma, principio que se encuentra incluso en nosotros, con el primer principio, centro de todo, nuestro "uno" con el Uno. Solo podemos ver el Uno-Bien haciéndonos "uno"; y esto solo es posible con lo que en nosotros hay de semejante a él, el "uno", centro del alma, ${ }^{43}$ por el que nos unimos a lo que nos es semejante, el Uno-Bien transcendente.

Como el primer principio está más allá de la Inteligencia, solo puede coincidir con aquello de la inteligencia que no es inteligencia: el centro del alma. Pero para ello el alma ha de despojarse de todo, quedarse a solas consigo misma, y desactivar los otros dos niveles del alma: el sensitivo-vegetativo y el intelectivo. En ese momento, partiendo de la imagen que hay en ella, es posible llegar al Uno-Bien. El vidente se reconoce como imagen del primer principio y pasa, a continuación, al modelo, transformándose en él, y llegando así al

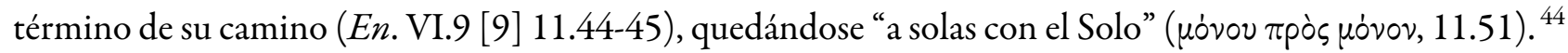
Se entiende de este modo que la belleza sea idóneamente el camino y la puerta que conduce al Uno-Bien. ${ }^{45}$

\section{El PROBLEMA DEL CONOCIMIENTO DEL PRIMER PRINCIPIO: CONTACTO}

¿Cuál es la naturaleza del ser inteligible con la que el alma está "emparentada”? 46 Para responder a esta cuestión, en el tratado Sobre la inmortalidad del alma, Plotino parte de una lectura (neo)platónica del Fedón de Platón para mostrar que "todo lo que es divino y bienaventurado" (

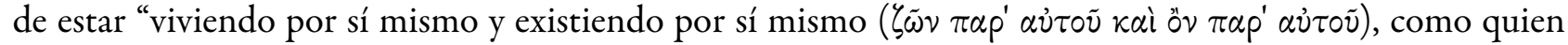

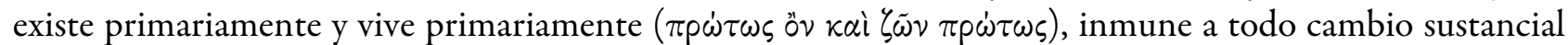

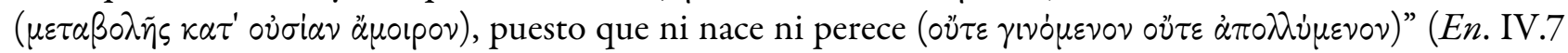
[2] 9.13-16). Plotino se inspira en la prueba de la inmortalidad del alma expuesta por Platón en el Fedro (245c5-246a2) y en la tercera del Fedón (102a-107b). Por su parte, replicando a esta tesis platónica, el peripatético Estratón de Lámpsaco (ca. 340/330-269/268 a.C.), sucesor de Teofrasto en la dirección del Liceo, consideraba que el alma es inmortal en tanto que existe, pero esto no implica que sea imperecedera. ${ }^{47}$ Plotino defiende la tesis platónica, enfrentándose a esta objeción peripatética, y propone regresar al último argumento del Fedón, pero ampliando su alcance: el alma no es solo esencialmente viviente, sino también

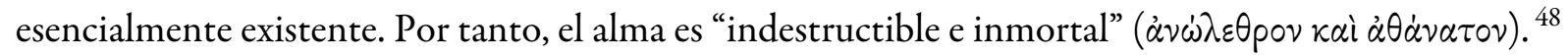

El alma es inmortal porque su naturaleza es la propia de un ser inteligible y divino. Precisamente, la arquitectura de la metafísica plotiniana permite concebir el alma siendo al mismo tiempo un ser derivado de los principios superiores -la Inteligencia y el Uno- y causado por sí mismo. Según el principio que Igal denomina de la "génesis bifásica" (1982: 31-32), en un primer momento (fase proódica), la actividad generada por el productor, al carecer de contenido, es aún indeterminada e informe; en un segundo momento (fase epistrófica), el término generado regresa a su progenitor, y así se configura y se lleva a cabo su propio perfeccionamiento (En. III.4 [15] 1). De este modo, el alma se perfecciona a sí misma cuando regresa epistróficamente al principio inteligible del que procede. 
Lo que es inteligible no deberá unas veces ser y otras no ser, y se distinguirá de la "cosa blanca", que no tiene el ser en su naturaleza propia, pues unas veces es blanca y otras no. ${ }^{49}$ Plotino contrapone el ser por sí, inteligible, emparentado y consustancial con las cosas divinas, ${ }^{50}$ a las cosas sensibles y materiales, que forman parte, en cuanto materiales, de lo mortal y perecedero. El alma participa de las cosas divinas.

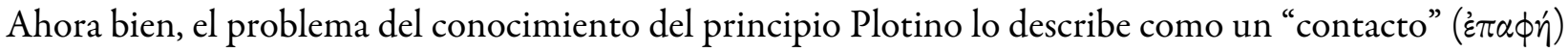
inefable con él semejante a la visión que el alma tiene de la luz del principio. De este modo, "un alma

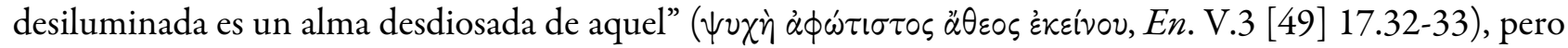
cuando es iluminada, obtiene lo que buscaba. El alma ve la luz del principio gracias a la propia luz del principio, es decir, ve con esa misma luz, no con la luz de otro, sino gracias a la misma luz por la que ella ve. ${ }^{51}$ Así pues, lo que el alma ve, su visión y la luz que le permite ver coinciden en un mismo punto. El alma, una vez que ha remontado a la Inteligencia, participa de la visión del primer principio que tiene la Inteligencia. Plotino

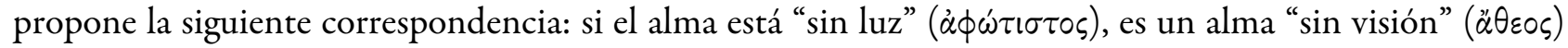
del Uno-Bien. ${ }^{52}$ Aquí, como sostiene Fronterotta (2009: 404), el término $\alpha \theta$ عos es un hapax en Plotino, y no puede otorgársele el sentido de $\grave{\alpha}-\theta \dot{\varepsilon} \alpha$, interpretando que el alma "sin luz" está, asimismo, "privada de esa visión”.

Para llegar al Uno-Bien el alma ha de liberarse de todo lo que represente un obstáculo, y así, despojada de todo (En. V.3 [49] 17.38), puede elevarse sola y pura hacia su principio (VI.9 [9] 9.11). Pero Plotino alerta sobre la insuficiencia del pensamiento discursivo ( $\delta \iota \dot{\alpha} v o ı \alpha$ ) para lograr el conocimiento de las realidades superiores. Asimismo, por lo que concierne al conocimiento del principio, establece una oposición entre el pensamiento ( $(o ́ n \sigma ı)$, que se caracteriza por un fraccionamiento del sujeto que piensa y del objeto que es pensado, y el contacto unitivo, ${ }^{53}$ que no guarda ningún tipo de relación con el pensamiento. ${ }^{54}$

De lo contrario, no habrá intelección (vónбıs) de ese objeto, sino solo una aprehensión ( $\theta i \xi ı$ ) y una especie

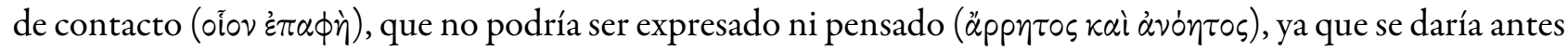

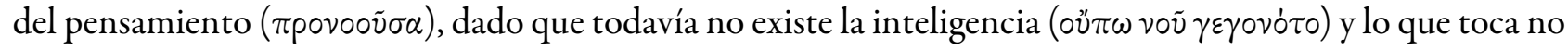

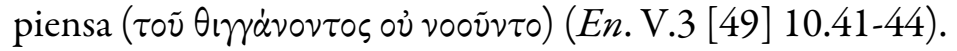

Ahora bien, mientras que sobre el conocimiento del principio, Plotino recurre a un contacto unitivo, Jámblico sostiene la imposibilidad del conocimiento del principio, ya que solo es posible alcanzar, por medio de una "conexión" ( $\sigma \nu v \alpha \phi \eta \dot{\eta}),{ }^{55}$ el conocimiento humano de los dioses, que son inferiores al principio. Según Jámblico, la inteligencia conoce a los dioses por “conexión”, mediante una "intelección innata” y mediante "intelecciones puras e irreprochables", recibidas de los dioses desde la eternidad. De este modo, para abordar el conocimiento humano de los dioses, Jámblico vincula estas entidades divinas, en cuanto objeto innato del conocimiento, con las intelecciones recibidas de los dioses. ${ }^{56}$ Esta conexión quedaría probada por la

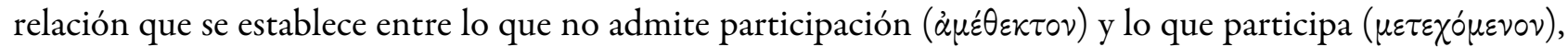
lo que introduce también la relación entre el "intelecto en acto", que posee la eternidad, la perfección y la permanencia, y el "intelecto en potencia", que posee una especie de receptividad pasiva respecto a las intelecciones. ${ }^{57}$ Por tanto, la perspectiva neoplatónica ha cambiado: para Plotino puede darse un contacto unitivo con el principio, y para Jámblico, en cambio, esta propuesta queda excluida del todo, ya que solo hay conexión con los dioses, pero no contacto con el principio.

\section{A MOdo De conclusión, El ÚlTimo consejo A EuSTOQuio}

Para Plotino la atención $(\pi \rho \circ \sigma \circ \chi \dot{\eta})$ es un estado de conciencia que implica una conversión al alma superior y separada, y a las huellas que deja en ella la Inteligencia (En. V.1 [10] 12.12-20). En este sentido, la atención se opone a la preocupación ( $\kappa \eta \delta \varepsilon \mu o v i ́ \alpha)$, que califica de "alboroto" ( $\theta o ́ p v \beta o s)$ (En. I.1 [53] 9.25), ya que implica una conciencia del todo atrapada por el cuerpo animado. Por ello, "mantener la facultad perceptiva

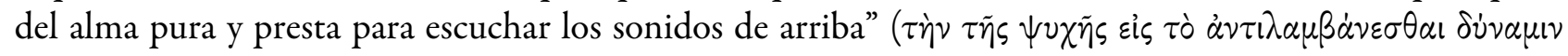




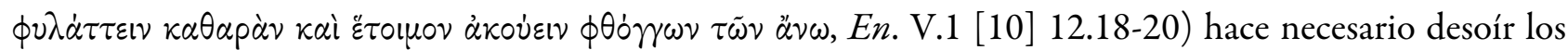
sonidos sensibles, ya que en el mundo sensible no puede haber verdadera comunidad ni darse una auténtica comunicación. Pero, forzado por la necesidad, puede dar un consejo, como las palabras que dirige a Eustoquio.

Del encuentro entre el pensamiento intelectivo y el tiempo resulta la diánoia. El alma, considerada en su relación con el cuerpo, viene definida por la discursividad, es decir, por el distanciamiento con respecto a la Inteligencia, que conlleva una distensión de la vida eterna. Por ello, Plotino considera el acto discursivo

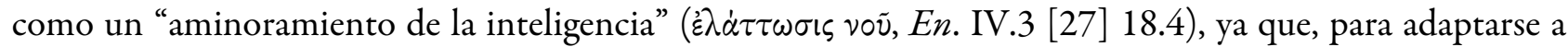
las exigencias del cuerpo y de la acción, despliega en momentos sucesivos la inmediatez y la totalidad de la intuición.

Como señala Aubry (2014: 285), "la experiencia mística plotiniana es contacto no con el Uno-Bien mismo sino con su potencia, es decir, con el punto de emanación de lo real". ${ }^{58}$ En este sentido, podemos interpretar las últimas palabras de Plotino en conexión con la invención de un nuevo modelo de causalidad. ${ }^{59}$ Por debajo del Uno-Bien, cada ser culmina su perfección y se actualiza por medio de la contemplación del ser que le supera: la Inteligencia en el Uno-Bien, el alma en la Inteligencia. Ahora bien, la naturaleza es la potencia inferior del alma del mundo. A diferencia del alma individual, la naturaleza no se vuelve a contemplar la Inteligencia por medio del cuerpo que dirige, sino que es el producto o la imagen de la contemplación del alma del mundo. Por ello el cuerpo del mundo, que es un kósmos, está mejor regido que los cuerpos individuales. La naturaleza es "una contemplación silenciosa, mas un tanto desvaída" ( $\theta \varepsilon \omega p i \alpha a ̈ \psi \circ \phi \circ \varsigma, ~ \alpha ُ \mu v \delta \rho \circ \tau \dot{p} \rho \alpha, E n$. III.8

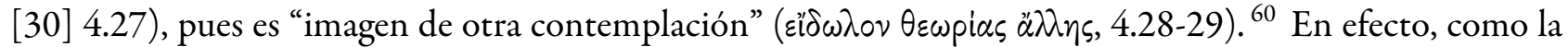
contemplación del alma de la que procede, no se dirige hacia lo que le es inferior, ni tampoco hacia lo que le es superior, sino al interior de sí misma, de donde deriva su fecundidad. Por ello, el auténtico regreso a la interioridad implica un hacer elevar el alma a lo divino por medio de "una contemplación productiva" que, ${ }^{61}$ al activar la mirada, culmina el esfuerzo de la anagogé.

\section{REFERENCIAS}

Armstrong, A. H. (1966) Plotinus: Enneads, vol. I, Cambridge, Mass.

Atkinson, M. (1983) Plotinus: Ennead V. 1. On the Three Principal Hypostases: a Commentary with Translation, Oxford.

Aubry, G. (2014) “'Comprendre et se taire’. Plotin et les discours du silence”, en L. Boulègue, P. Caye, F. Malhomme \& S. Perceau (eds.) Silence et sagesse. De la musique à la métaphysique: les anciens Grecset leur héritage, Paris: 273-291.

Beierwaltes, W. (1967) Plotin. Über Ewigkeit und Zeit: (Enneade III, 7), Frankfurt.

Beierwaltes, W. (1985) Denken des Einen. Studien zum Neuplatonismus und dessen Wirkungsgeschichte, Frankfurt.

Beierwaltes, W. (2004 ${ }^{2}$ [1972]) Platonismus und Idealismus, Frankfurt.

Beierwaltes, W. (2013) "Plotins Theorie des Schönen und der Kunst", en F. Karfík \& E. Song (eds.) Plato Revived. Essays on Ancient Platonism in Honour of Dominic J. O'Meara, Berlin-Boston: 3-26.

Blumenthal, H. J. (1971) Plotinus' Psychology: His Doctrines of the Embodied Soul, The Hague.

Bregman, J. (2010) "Synesius of Cyrene”, en L. P. Gerson (ed.) The Cambridge History of Philosophy in Late Antiquity, vol. I, Cambridge: 520-537.

Brisson, L. (1990) “L'oracle d'Apollon dans la Vie de Plotin par Porphyre”, Kernos 3: 77-88.

Brisson, L. (2010) "Porphyre. Sur la vie de Plotin et la mise en ordre de ses livres", en L. Brisson \& J.-F. Pradeau (eds.) Plotin: Traités 51-54. Porphyre: Vie de Plotin, Paris: 287-335.

Brisson, L. et al. (eds.) (1992) Porphyre: Vie de Plotin, vol. II, Paris.

Brisson, L. et al. (2005) Porphyre: Sentences, 2 vols., Paris.

Burnet, J. (1900-1907) Platonis Opera, 4 vols., Oxford. 
Burnyeat, M. F. (2005) “Eikōs Muthos”, Rhizai 2: 143-165.

Cano Cuenca, J. (2012) “Eikos logos-eikos mythos: un logos como representación del mundo”, Revista de Estudios Sociales 44: 36-47.

Chiaradonna, R. (2015) “Plotin lecteur du Phédon: l'âme et la vie en IV 7 [2] 11”, en S. Delcomminette, P. D’Hoine \& M.-A. Gavray (eds.) Ancient Readings of Plato's Phaedo, Leiden: 154-172.

D’Ancona Costa, C. (2002) “'To Bring Back the Divine in Us to the Divine in the All'. Vita Plotini 2, 26-27 Once Again”, en T. Kobusch \& M. Erler (eds.) Metaphysik und Religion: zur Signatur des spätantiken Denkens: Akten des internationalen Kongresses vom 13.-17. März 2001 in Würzburg, München: 517-565.

De Rijk, L. M. (1990) “Causation and Participation in Proclus. The Pivotal Role of Scope Distinction”, en A. P. Bos \& P. A. Meijer (eds.) On Proclus and his Influence in Medieval Philosophy, Leiden-New York-Köln: 1-34.

Des Places, E. (1966) Jamblique: Les Mystères d'Égypte, Paris.

Des Places, E. (1989) Jamblique: Protreptique, Paris.

Diggle, J. (1984) Euripidis Fabulae: Cyclops. Alcestis. Medea. Heraclidae. Hippolytus. Andromacha. Hecuba, Tomus I, Oxford.

Dodds, E. R. (1963² [1933]) Proclus: The Elements of Theology, Oxford.

Dodds, E. R. (1965) “Reviewed Work: T. 1: Porphyrii Vita Plotini. Enneades 1-3 by Plotini Opera, Paul Henry, HansRudolf Schwyzer”, Gnomon 37: 419-420.

Fronterotta, F. (2009) "Traité 49 (V, 3). Sur les hypostases qui connaissent et sur ce qui est au-delà. Présentation, traduction et notes", en L. Brisson \& J.-F. Pradeau (eds.) Plotin: Traités 45-50, Paris: 301-405.

García Bazán, F. (2005) "Plotino y la fenomenología de la belleza”, ASHF 22: 7-28.

García Romero, F. (1995) Sinesio de Cirene: Cartas, Madrid.

Garzya, A. \& Roques, D. (2000) Synésios de Cyrène: Correspondance, 2 vols., Paris.

Giangrande, G. (1956) Eunapii Vitae sophistarum, Roma.

Goulet, R. (1982) “L'Oracle d'Apollon dans la Vie de Plotin", en L. Brisson, M.-O. Goulet-Cazé, R. Goulet \& D. O’Brien (eds.) Porphyre: La Vie de Plotin. Travaux préliminaires et index grec complet, vol. I, Paris: 378-379.

Goulet-Cazé, M.-O. (1982) “L’Édition porphyrienne des Ennéades: État de la question”, en L. Brisson, M.-O. GouletCazé, R. Goulet \& D. O’Brien (eds.) Porphyre: La Vie de Plotin. Travaux préliminaires et index grec complet, vol. I, Paris: 280-327.

Grmek, M. D. (1992) “Les maladies et la mort de Plotin”, en L. Brisson et al. (eds.) Porphyre: la Vie de Plotin, vol. II, Paris: 335-353.

Hadot, P. (1983) "Physique et poésie dans le Timée de Platon", RThPh 115: 113-133. (= Reimpr. 1998, Études de philosophie ancienne, Paris: 277-305).

Hadot, P. (1994) Plotin: Traité 9 (Ennéade VI, 9), Paris.

Harder, R. (1958) Porphyrios: Über Plotins Leben und über die Ordnung seiner Schriften (=Plotins Schriften), Bd. Vc, Hamburg.

Henry, P. (1953) “La dernière parole de Plotin”, SCO 2: 113-130.

Henry, P. \& Schwyzer, H.-R. (1951-1973) Plotini opera, 3 vols., Paris-Bruxelles-Leiden [= editio maior, H-S ${ }^{1}$.

Henry, P. \& Schwyzer, H.-R. (1964-1982) Plotini opera, 3 vols., Oxford [= editio minor, H-S ${ }^{2}$.

Igal, J. (1972) “Una nueva interpretación de las últimas palabras de Plotino”, CFC 4: 441-462.

Igal, J. (1982) Porfirio: Vida de Plotino. Plotino: Enéadas I-II, Madrid.

Lewy, H. (2011 ${ }^{3}$ [1956]) Chaldaean Oracles and Theurgy: Mystic Magic and Platonism in the Later Roman Empire, Troisième édition par M. Tardieu avec un supplément "Les Oracles chaldaïques 1891-2011", Paris.

Marcovich, M. (2001) Origenes: Contra Celsum. Libri VIII, Leiden.

Mayhoff, C. (1892-1909 [1967]) C. Plini Secundi naturalis historiae, 5 vols., Leipzig. 
Mazur, Z. (2017) “To Try to Bring the Divine in Us Back Up to the Divine in the All': The Gnostic Background of Plotinus's Last Words", JECS 25: 561-580.

Meijer, P. A. (1992) Plotinus on the Good or the One (Enneads VI, 9): an Analytical Commentary, Amsterdam.

Monat, P. (1992-1997) Firmicus Maternus: Mathesis, 3 vols., Paris.

Most, G. W. (2003) “Plotinus' Last Words”, CQ 53: 576-587.

Narbonne, J.-M., Archard, M. \& Ferroni, L. (2014) Plotin: CEuvres complètes, I, 1. Introduction générale, Traité 1: Sur le Beau, Paris.

O’Brien, D. (1997) “Immortal and Necessary Being in Plato and Plotinus", en J. J. Cleary (ed.) The Perennial Tradition of Neoplatonism, Leuven: 39-103.

O’Meara, D. J. (1996) “Textes de Plotin sur la beauté: initiation et remarques”, en I. Schussler, R. Celis \& A. Schild (eds.) Art et verité, Lausanne: 59-68.

Pépin, J. (1992) "La dernière parole de Plotin (VP 2.23-27)", en L. Brisson et al. (eds.) Porphyre: la Vie de Plotin, vol. II, Paris: 355-383.

Perna, P. (1580) Plotini Opera omnia. Cum latina Marsilii Ficini interpretatione et commentatione, Basilea.

Peterson, E. (1933) “Herkunft und Bedeutung der MONO $П$ ПО $\Sigma$ MONON-Formel bei Plotin”, Philologus 88: $30-41$.

Ramos Jurado, E. A. (1989) Pseudo Plutarco: Sobre la vida y poesía de Homero. Porfirio: El antro de las ninfas de la Odisea. Salustio: Sobre los dioses y el mundo, Madrid.

Rochefort, G. (1960) Saloustios: Des dieux et du monde, Paris.

Rodríguez Adrados, F. (1980-2010) Diccionario griego-español (DGE), 7 vols., Madrid.

Rose, V. (1886) Aristotelis qui ferebantur librorum fragmenta, Leipzig.

Ross, W. D. (1955) Aristotelis fragmenta selecta, Oxford.

Ruiz de Elvira Prieto, A. (1982-1984²) Ovidio: Metamorfosis, 3 vols., Madrid.

Sala, T. A. (2002) "Die entwendeten (vor)letzten Worte Plotins", Prima Philosophia 15: 327-342.

Santa Cruz, M. I. (2006) “Modos de conocimiento en Plotino”, Estudios de Filosofía 34: 201-216.

Schwyzer, H.-R. (1976) “Plotins letztes Wort”, $M H$ 33: 85-97.

Sedley, D. (1997) "Becoming Like God in the Timaeus and Aristotle", en T. Calvo \& L. Brisson (eds.) Interpreting the Timaeus-Critias. Proceedings of the IV Symposium Platonicum, Sankt Augustin: 327-339.

Sedley, D. (1999) “The Idea of Godlikeness”, en G. Fine (ed.) Plato 2: Ethics, Politics, Religion, and the Soul, Oxford: 309-328.

Sharples, R. W. (2011) "Strato of Lampsacus: The Sources, Texts and Translations", en M. L. Desclos \& W. W. Fortenbaugh (eds.) Strato of Lampsacus: Text, Translation, and Discussion, New Brunswick: 5-229.

Smith, W. D. (1990) Hippocrates: Pseudepigraphic Writings, Leiden.

Taormina, D. P. (1999) Jamblique, critique de Plotin et de Porphyre. Quatre études, Paris.

Toulouse, S. (2016) "Synésios de Cyrène", en R. Goulet (ed.) Dictionnaire des philosophes antiques, vol. VI, Paris: 639-676.

Trouillard, J. (1955) La purification plotinienne, Paris.

Vidart, T. (2006) “Il faut s'enfuir d'ici': la relation de l'homme au monde", EPlaton 3: 141-152.

Wachsmuth, C. \& Hense, O. (1884-1912) Ioannis Stobaei Anthologium, 5 vols., Berlin.

Walzer, R. R. \& Mingay, J. M. (1991) Aristotelis Ethica Eudemia, Oxford.

Westerink, L. G. (1977) The Greek Commentaries on Plato's Phaedo, vol. 2, Damascius, Amsterdam-Oxford-New York.

Zamora Calvo, J. M. (2017) "Beauty, Beautiful, and Beautifulness in Plotinus (Enn. I, 6 [1] 6, 13-32)”, Eirene 53: 127-149. 


\section{Notas}

1. Cf. Porfirio, V.P. 2.19. De Zeto, que era médico, conocemos lo siguiente: "Plotino le profesaba un amor profundo ( $\sigma \phi \delta \delta \rho \alpha \pi \varepsilon \phi i \lambda \omega \tau \circ)$; mas, como seguía la carrera política y abrigaba inclinaciones políticas, Plotino procuraba refrenarlo. Trataba con él familiarmente (oikei $\omega \varsigma$ ), tanto que solía retirarse a su finca, situada a seis millas de Minturnas”. Pofririo, V.P. 7.19-24; trad. Igal (1982: 142-143). Este trabajo se enmarca dentro del Proyecto I+D: "Lecturas neoplatónicas sobre la inmortalidad del alma: de Plotino a Damascio" (Ref. HAR2017-83613-C2-2-P) y de las actividades del Grupo de Investigación de la UAM: "Influencias de las éticas griegas en la filosofía contemporánea" (Ref. F-055).

2. Dos fuentes nos informan sobre las enfermedades que padeció Plotino y sobre las circunstancias de su muerte: la biografía de su discípulo Porfirio (V.P. 2.10-15), escrita treinta años después de su fallecimiento, y el tratado astrológico de Julio Fírmico Materno, intitulado Máthesis (I.7.14-22), redactado en torno a 330. Según Grmek (1992: 353), el diagnóstico retrospectivo de lepra no está justificado. Las manifestaciones que señala Porfirio corresponden más bien a un caso de tuberculosis, o a una gangrena, pudiendo considerarse también el ergotismo (intoxicación con cornezuelo de centeno) o la diabetes.

3. Porfirio menciona los nombres de los miembros que componían este círculo de "discípulos" ( $\gamma \nu \omega \dot{p} \iota \mu o \iota)$, amigos y

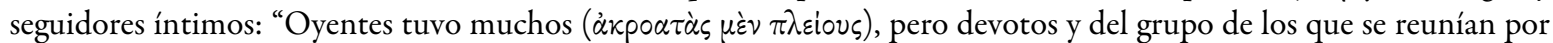

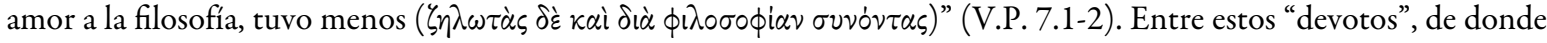
quedan excluidos sus "amigos” los gnósticos, Porfirio menciona a Amelio Gentiliano, nacido en Etruria, asistente de Plotino (18.8-23); Paulino de Escitópolis, apodado por Amelio "Mícalo”, porque, a su juicio, estaba lleno de doctrinas mal entendidas (7.7); el médico Eustoquio de Alejandría (2.23-24); el crítico y poeta Zótico, quien puso en verso el relato de la Atlántida extraído del Critias platónico; el árabe Zeto, también médico, a cuya finca en Campania se retiraba frecuentemente Plotino; y Castricio Firmo (7.24-29). Entre sus “oyentes" se encontraban los senadores Sabinilio y Marcelo Orroncio, así como Rogaciano, quien renunció a su cargo y a todas sus posesiones por seguir la filosofía; el retórico Serapión de Alejandría, que también asistía a los cursos de Plotino, por el contrario, no abandonó sus actividades de prestamista ni su debilidad por el dinero. Entre estos compañeros estaba también el propio Porfirio de Tiro, quien fue el encargado de corregir y editar los escritos de su maestro (7.29-51).

4. Cf. Igal (1982: 8, n. 2). Los últimos días de Plotino coinciden con el final del segundo año del reinado de Claudio; cf. Porfirio, V.P. 2.34-37.

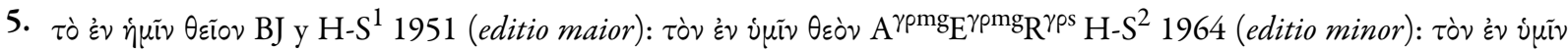
$\theta \varepsilon \tilde{o} 0 \mathrm{w}$ w.

6. B (Laurentianus 85, 15), códice del s. XIV, perteneciente a la familia x hasta En. IV.9 [8] 3.6; J (Parisinus Graecus 2082), códice del s. XV; Familia y: U (Vaticanus Urbinas Graecus 62), del s. XV; S (Berolinensis Graecus 375), del s. XV; y C (Monacensis Graecus 449, del s. XV).

7. Cf. Platón, Timeo 90c; Aristóteles, Protréptico fr. 61 Rose = 10c Ross; Ética eudemia VIII.2.1248a27.

8. A (Laurentianus 87, 3), códice del s. XIII; E (Parisinus Graecus 1976), del s. XIII; R (Vaticanus Reginensis Graecus 97), del s. XIV. Los códices A y E pertenecen a la familia w, y el códice R a la familia x. Los 58 códices que conservamos de las Enéadas proceden de un arquetipo común, que no ha llegado hasta nosotros, redactado probablemente entre los s. IX y XII.

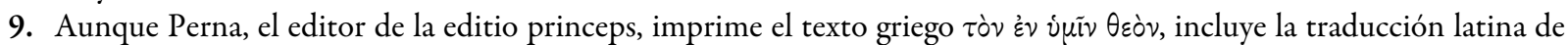
Ficino "quod in nobis diuinum est", que conserva la interpretación tradicional. Cf. Henry (1953: 116-117).

10. Cf. H-S ${ }^{1}$ (1973: 348), vol. III, Addenda ad textum.

11. Armstrong (1966: 6-7) incluye el texto griego de Henry (1953), pero admite la posibilidad de adoptar la corrección que propone de Strycker: $\pi \varepsilon \iota \rho \tilde{\alpha} \sigma \theta \varepsilon$ en lugar de $\pi \varepsilon \iota p \tilde{\alpha} \sigma \theta \alpha$.

12. Cf. Henry (1953: 130): "Efforcez-vouz de faire remonter le Dieu qui est en vous jusqu'au divin qui est dans l'univers".

13. Además de estas variantes, el códice del siglo XIV: R (Vaticanus Reginensis Graecus 97), incluye el siguiente texto: $\tau \grave{\nu} v$

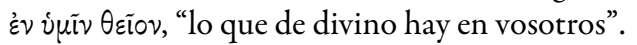

14. Probablemente existía una edición anterior de los escritos de Plotino a la elaborada por Porfirio. Un escolio conservado a En. IV.4 [28] 29.55 nos permite suponer que Eustoquio fue el autor de esta edición anterior, a la que Porfirio sin embargo no hace mención alguna: "Hasta aquí había en los manuscritos de Eustoquio el segundo tratado Acerca del alma y en ese lugar comenzaba el tercer tratado. En los manuscritos de Porfirio, en cambio, lo que sigue pertenece al segundo tratado". Cf. Henry (1935) y Goulet-Caze (1982: 287-294).

15. Cf. Brisson (1992: 203).

16. Cf. Hipócrates, Epistolas 15.

17. Cf. Plinio el Viejo, Historial natural X.188; Ovidio, Metamorfosis XV.389-390; Orígenes, Contra Celso IV.57.

18. Cf. Eurípides, Hécuba 571.

19. Sobre este oráculo, véase Goulet (1982); Brisson (1990). 
20. Cf. Sinesio, Cartas 41. 331-334: "Pero, lo mismo que no he sido un filósofo corriente ni he buscado el aplauso en los teatros ni he abierto una escuela (aunque, por eso, no era yo menos filósofo y, de hecho, desearía serlo aún), así tampoco quiero ser un sacerdote corriente". Cf. Toulouse (2016: 639-640).

21. Cf. García Romero (1995: 11-12; 325-326).

22. Entre los filólogos y comentaristas hay discrepancias sobre el carácter relevante o no de una posible conexión precisa entre el testimonio de Porfirio y la carta de Sinesio. Cf. Henry (1953: 126-130, n. 3); Harder (1958: 81, n. 7); Igal (1972: 453-457, n. 4); Schwyzer (1976: 89-90, n. 1); Pépin (1992: 375-376, n. 6). Para su redacción, Most (2003: 582-283, n. 28-31) no duda de que Sinesio tiene en su cabeza el pasaje concreto de la Vida de Plotino (2.23-29), considerando que el

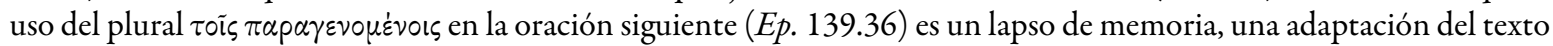
a sus exigencias retóricas concretas, una inclusión del pasaje en el entorno doméstico de la villa en la que reside Plotino, o una generalización que va más allá de Eustoquio, su único destinatario inmediato, al poder remitirse a toda la humanidad.

23. Porfirio, a la manera de Plotino, no respeta siempre la diferencia entre Alma hipóstasis y alma del mundo; cf. Blumenthal (1971).

24. En su Elogio de la calvicie, Siriano menciona una tradición que propone lo siguiente: "el alma quiere imitar el dios (cf. Platón, Timeo 33b-36b), es decir, el tercer dios, el alma del mundo, que su padre, el demiurgo del mundo físico, ha introducido en el mundo, haciéndole así perfecto y total y haciendo de él un todo compuesto de todas las semillas y de todos los cuerpos" (VIII.205.1.5-10). Cf. Garzya y Roques (2000: 403, n. 22).

25. Hierocles exhorta a ponerse los unos en el lugar de los otros y a abrirse progresivamente en círculos concéntricos a la humanidad, pero sin demoler, desde el centro de la razón, la perspectiva de lo propio y familiar. Cf. Hierocles, apud Estobeo, Eclogae 4.671.7-673.11.

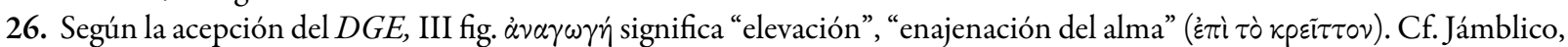
Sobre los misterios egipcios 3.7; Porfirio, Sentencias 30; Eunapio, Vidas de filósofos y sofistas 482. Asimismo, los caldeos habrían tomado prestado el término $\alpha \nu \alpha \gamma \omega \gamma \eta \dot{~ d e l ~ v o c a b u l a r i o ~ q u e ~ d e s c r i b e ~ l a ~ a p o t e o s i s ~ d e ~ l o s ~ h e ́ r o e s . ~ S o b r e ~ e l ~ t e ́ r m i n o ~}$ $\dot{\alpha} \nu \alpha \gamma \omega \gamma \dot{\eta}$, véase Lewy $\left(2011^{3}\right.$ [1956]: 488-491).

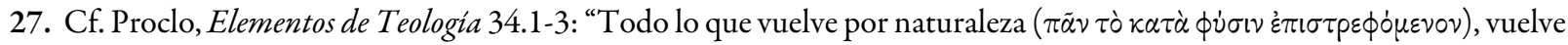

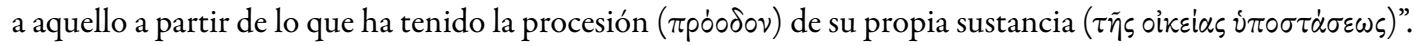

28. Cf. Plotino, En. I.1 [53] 12.24-25; IV.8 [6] 5.16-21. Trouillard (1955: 202) señala la siguiente diferencia entre la "falta" plotiniana y el "pecado" cristiano: mientras que el pecado en el cristianismo es "revuelta de espíritu" (el pecado del hombre es orgullo y desafío), en Plotino la "falta" es "debilidad del alma". Y, a su vez, frente a la idea cristiana de "redención" se halla la idea neoplatónica de "purificación".

29. Plotino califica la materia de primer mal para el alma (En. I.8 [51] 14.50-51). Ahora bien, el mal moral procede de la relación que se establece entre el alma y la materia, ya que la materia no constituye el mal moral.

30. Cf. Platón, República VII.532e3.

31. Cf. Igal (1972: 451).

32. Cf. Santa Cruz (2006: 208).

33. Cf. Narbonne, Archard y Ferroni (2014: cccxvIII-CCCXIX).

34. Cf. Vidart (2006: 150-151).

35. Cf. O’Meara (1996: 61); Zamora Calvo (2017: 129-132).

36. Cf. Igal (1982: 287, n. 42).

37. Cf. Hadot (1983: 129-131); Burnyeat (2005: 158-159); Cano Cuenca (2012: 39-40).

38. Cf. Sedley (1997: 327-329; 1999: 309-310).

39. Cf. Platón, Filebo 20d1; 54c10; 60b4. Cf. Narbonne, Archard y Ferroni (2014: 58, n. 2).

40. Sobre la teoría plotiniana de la visión, véase Atkinson (1983: 18-21).

41. Cf. Platón, República VI.507c; 508b; 509a.

42. Cf. Plotino, En. III.8 [30] 9.22-23; V.1 [10] 11.13; VI.9 [9] 3.20-21; 8.19-20.

43. Sobre el "centro del alma", principio interior, que coincide con el primer principio transcendente, véase Igal (1982: 88-89 y 98-99); Hadot (1994: 210-216).

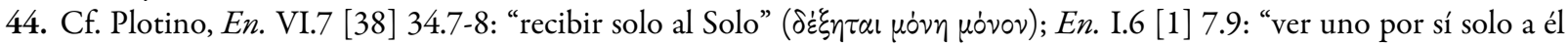

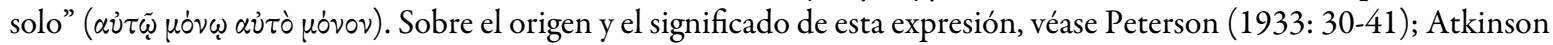
(1983: 131-132); Meijer (1992: 157-162); Hadot (1994: 51-53); Beierwaltes (2013: 15-16).

45. Cf. García Bazán (2005: 16-18).

46. Cf. Platón, República 611e2-3.

47. Cf. Damascio, Comentario al Fedón de Platón I.431-443 = Estratón, fr. 80. Cf. Sharples (2011: 171-175).

48. Cf. Plotino, En. IV.7 [2] 9.11. Cf. O’Brien (1997: 57-60); Chiaradonna (2015: 157-159).

49. Cf. Plotino, En. IV.7 [2] 9.18-21.

50. Cf. Plotino, En. IV.7 [2] 10.18-19. 
51. Cf. Plotino, En. V.3 [49] 17.34-36.

52. Cf. $D G E: 2 \ddot{\alpha} \theta \varepsilon \circ \varsigma,-\circ \nu$, segunda acepción.

53. Sobre la interpretación de દ̇ $\pi \alpha \phi \eta ́$ como “contacto unitivo", véase Taormina (1999: 152-154).

54. Cf. Plotino, En. VI.7 [38] 39.19-20.

55. En el Protréptico (49.17-18), Jámblico aborda la superioridad de la inteligencia sobre el alma, y considera la "conexión" ( $\sigma \nu \alpha \phi \dot{\eta})$ y la pureza de las intelecciones como características fundamentales de la actividad mediante la cual la inteligencia conoce a los dioses. Cf. Jámblico, Protréptico 21; Sobre los misterios egipcios I.12; V.3; V.21; V.22; V.26.

56. Cf. Taormina (1999: 52).

57. Proclo describe en términos de procesión y de irradiación el proceso descendente que sigue el orden jerárquico desde

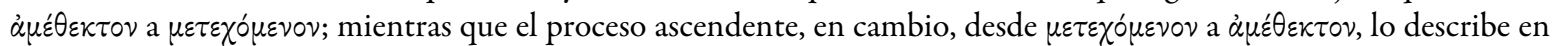
términos de semejanza y de participación. Cf. De Rijk (1990: 8-9).

58. Cf. Plotino, En. VI.9 [9] 4.34-30.

59. Cf. Salustio, Sobre los dioses y el mundo 16.1.8-12; trad de Ramos Jurado (1989: 307-308): “Además, la felicidad de

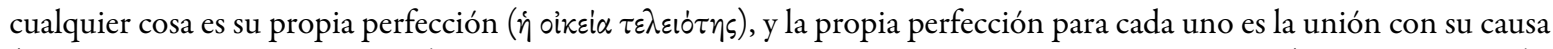

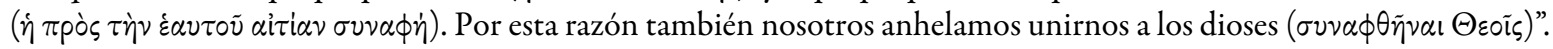
Cf. Porfirio, Sobre la abstinencia II.34; Jámblico, Sobre los misterios egipcios III.20.27.

60. Cf. Plotino, En. IV.4 [28] 13.

61. Cf. Beierwaltes (2004² [1972]: 139).

* Profesor titular de Filosofía Antigua en la Universidad Autónoma de Madrid. Doctor en Filosofía, con premio extraordinario, por la Universidad de Valladolid (1998). Ha realizado estancias de investigación en la Universidad Libre de Bruselas, el CNRS y el CONICET. Es presidente de la Sección española de la International Society for Neoplatonic Studies, miembro asociado del Centro de Filosofía de la Universidad de Lisboa, director del Grupo de investigación "Influencias de las éticas griegas en la filosofía contemporánea" (Ref. F-055) y coordinador del Máster universitario en "Filosofía de la historia: Democracia y orden mundial". ORCID ID: orcid.org/0000-0001-7101-2234. 